\title{
Viability of Sea Fan Populations Impacted by Disease: Recruitment versus Incidence
}

\author{
Alberto M. Sabat ${ }^{1}$ and Carlos Toledo-Hernández ${ }^{2}$ \\ ${ }^{1}$ Department of Biology, University of Puerto Rico, San Juan, PR 00931-3360, USA \\ ${ }^{2}$ Sociedad Ambiente Marino, P.O. Box 22158, San Juan, PR 00931-2158, USA \\ Correspondence should be addressed to Alberto M. Sabat; amsabat@gmail.com
}

Received 24 September 2014; Revised 29 April 2015; Accepted 29 April 2015

Academic Editor: Baruch Rinkevich

Copyright (C) 2015 A. M. Sabat and C. Toledo-Hernández. This is an open access article distributed under the Creative Commons Attribution License, which permits unrestricted use, distribution, and reproduction in any medium, provided the original work is properly cited.

\begin{abstract}
Diseases are a threat to the viability of an increasing number of coral populations. In this study we analyze the effect of infection and recruitment rate on the viability and structure of sea fan (Gorgonia ventalina) populations using a size-based matrix model parameterized with data from field studies. The model predicts that the viability of sea fan populations is strongly influenced by disease incidence and recruitment. Under high recruitment rate, the disease incidence threshold for population viability is $0.12 / \mathrm{yr}$. However, populations with no or low incidence may also go locally extinct given persistent low recruitment. The model also predicts an effect of recruitment on disease prevalence. Everything else being equal, sites with low recruitment will exhibit higher disease prevalence than ones with high recruitment, particularly in medium and large colonies. Elasticity analysis reveals that changes in colony survivorship are likely to have the strongest effect on population growth rate, particularly given low recruitment. We conclude that under current levels of incidence sea fan populations in the Caribbean are not at risk. However, future epizootics are likely to produce local extinctions particularly if coinciding spatially or temporally with low recruitment.
\end{abstract}

\section{Introduction}

Temporal variability in the abundance of sessile marine invertebrates at a locality equals the difference between number of larvae successfully settling during a given time period (i.e., recruitment) and the number of previously recruited individuals or colonies dying during the same time period [1]. Thus, population viability depends on the net effect between processes that affect recruitment [2] and those impinging on the mortality of the already established colonies. An increasing number of coral populations are being threatened by colony mortality due to diseases $[3,4]$, particularly in the Caribbean where at least 20 diseases affecting no less than 55 scleractinian and octocoral species have been reported [5]. Yet, for the most part, coral disease studies measure disease prevalence and/or tissue loss due to lesions per unit area and not the type of data that would be necessary to demographically assess the impact of disease on population viability.

A coral population visibly impacted by disease (i.e., high disease prevalence) may be at steady state or even exhibit growth, if recruitment rate equals or exceeds mortality rates of already established colonies, whereas an apparently healthy population (i.e., low disease prevalence) but with negligible recruitment during a long period may go locally extinct. In order to quantitatively discern the fate of coral populations impacted by disease we need (1) longitudinal studies in which the fate of diseased and healthy marked colonies is followed through time $[6,7]$ and (2) studies that model the effect of disease on the dynamics of coral populations [8-10].

Colonies of the sea fan Gorgonia ventalina are susceptible to a disease that has been attributed to the saprophytic fungus Aspergillus sydowii ([11, 12], however see [13, 14]). Die-offs of sea fans occurring in the subtropical and tropical Atlantic during the early 1990s were attributed to epizootics of this disease $[11,15,16]$. Although more recent studies indicate lower incidence $[6,17]$, the extent to which this affliction presents a current or future threat to the viability of sea fan populations in the Caribbean has only recently started to be addressed in a quantitative manner [10]. 
In this study, we use a size-based matrix population model [18] to analyze the viability of sea fan populations as a function of disease incidence and recruitment rate. We also analyzed the effect of disease incidence on the sea fan population structure, including disease prevalence (i.e., the proportion of diseased colonies in the different size classes) and the elasticity of life cycle transitions to population growth rate. Although the model was constructed and parameterized for $G$. ventalina, the approach is comprehensive and simple enough that given the availability of data on recruitment and the effect of a disease on the vital rates of a coral population, it can be used as a framework to assess the viability of populations of other coral species impacted by disease.

\section{Methods}

2.1. Model. We divided sea fans into three size classes based on surface area of live tissue, small $\left(<500 \mathrm{~cm}^{2}\right)$, medium $\left(500 \leq x \leq 1,000 \mathrm{~cm}^{2}\right)$, and large $\left(>1,000 \mathrm{~cm}^{2}\right)$, and two health categories, healthy and diseased. Size classes and health states follow those of Toledo-Hernández et al. [7] with the exception that we pooled data from "purpled colonies" (i.e., colonies with purple spots but no tissue necrosis) with that of healthy ones, as data from Toledo-Hernández et al. [7] indicates that survival and growth of purpled colonies are not different from those of healthy ones.

The number of colonies in each of the six life cycle stages, small healthy $\left(S_{h}\right)$, small diseased $\left(S_{d}\right)$, medium healthy $\left(M_{h}\right)$, medium diseased $\left(M_{d}\right)$, large healthy $\left(L_{h}\right)$, and large diseased $\left(L_{d}\right)$, at $t+1$ (one year) equals

$$
\begin{aligned}
&\left(\begin{array}{c}
S_{h} \\
S_{d} \\
M_{h} \\
M_{d} \\
L_{h} \\
L_{d}
\end{array}\right){ }_{t+1} \\
& \\
&\left(\begin{array}{ccccccc}
P 1_{h h} & P 1_{h d} & F 2+R 2_{h h} & R 2_{h d} & F 3^{2} & 0 \\
P 1_{d h} & P 1_{d d} & R 2_{d h} & R 2_{d d} & 0 & 0 \\
G 1_{h h} & 0 & P 2_{h h} & P 2_{h d} & R 3_{h h} & R 3_{h d} \\
G 1_{d h} & 0 & P 2_{d h} & P 2_{d d} & R 3_{d h} & R 3_{d d} \\
0 & 0 & G 2_{h h} & G 2_{h d} & P 3_{h h} & P 3_{h d} \\
0 & 0 & G 2_{d h} & G 2_{d d} & P 3_{d h} & P 3_{d d}
\end{array}\right)
\end{aligned}
$$

$$
\cdot\left(\begin{array}{c}
S_{h} \\
S_{d} \\
M_{h} \\
M_{d} \\
L_{h} \\
L_{d}
\end{array}\right)_{t}
$$

The contribution of each life cycle stage at time $t$ to all others at $t+1$ (one year) is given in the $6 \times 6$ matrix that projects the population vector between $t$ and $t+1$. $P 1, P 2$, and $P 3$ are the probabilities of small, medium, and large colonies, respectively, of remaining alive in the same size class. The subscripts $h h, h d, d h$, and $d d$ are, respectively, the annual probability of a healthy colony remaining healthy, of a diseased one healing, of a healthy one acquiring disease, and of a diseased remaining diseased. Thus, $P 1_{h h}$ is the probability that a small healthy colony will remain small and healthy for one year. The G's represent growth transitions. The $R$ 's are size retrogressions (i.e., becoming smaller by losing tissue either through disease-caused necrosis or by physical breakage). Finally, the $F$ 's are the contribution of recruits by local adults through sexual reproduction ( $G$. ventalina does not reproduce asexually). Notice that (1) diseased colonies do not reproduce [19], (2) medium healthy colonies contribute to small healthy ones via recruitment $(F 2)$ and via retrogression $\left(R 2_{h h}\right)$, and (3) small diseased colonies do not grow into medium-sized ones [7]. Equation (1) also assumes a closed population (i.e., the contribution of recruits from other populations is deemed negligible) and that colonies are infected only after settlement.

We calculated the real dominant eigenvalue of the projection matrix and its corresponding right and left eigenvectors to obtain the asymptotic growth rate of the population $(\lambda)$, the stable size structure $(\mathbf{w})$, the reproductive value vector $(\mathbf{v})$, and the resulting elasticity matrix [18]. We calculated $\lambda$ for different recruitment and disease incidence rates to explore the relationship between these variables. We also evaluate the effect of both recruitment and disease incidence on the population size structure including diseases prevalence. The elasticity analysis was performed because it offers insights as to which life cycle transitions should have more effect on $\lambda$ under different disease incidence and recruitment level scenarios.

2.2. Parameterization. If $\sigma$ is annual colony survivorship, $\alpha$ annual probability of acquiring disease (i.e., incidence), $\beta$ annual probability of healing, $\gamma$ annual probability of growing into the next size class, and $\rho$ the annual probability of retrogressing (i.e., becoming smaller due to tissue loss), the conditional probabilities of the life cycle states transitions are

$$
\begin{aligned}
G_{h h} & =\sigma \gamma(1-\alpha), \\
R_{h h} & =\sigma \rho(1-\alpha), \\
P_{h h} & =\sigma(1-\gamma)(1-\alpha)(1-\rho), \\
G_{d h} & =\sigma \gamma \alpha, \\
R_{d h} & =\sigma \rho \alpha, \\
P_{d h} & =\sigma \alpha(1-\gamma)(1-\rho), \\
G_{h d} & =\sigma \gamma \beta, \\
R_{h d} & =\sigma \rho \beta, \\
P_{h d} & =\sigma \beta(1-\gamma)(1-\rho),
\end{aligned}
$$




$$
\begin{aligned}
& G_{d d}=\sigma \gamma(1-\beta), \\
& R_{d d}=\sigma \rho(1-\beta), \\
& P_{d d}=\sigma(1-\gamma)(1-\beta)(1-\rho) .
\end{aligned}
$$

Annual survivorship was estimated by determining the fate of 92 healthy and diseased marked colonies of different size categories for one year [7]. Survivorship was found to be size dependent, with the main cause of colony mortality being detachment, which occurred in all size classes and health states, followed by disease, which was only observed in small fans that lost $100 \%$ of their live tissue due to disease. The data of Toledo-Hernández et al. [7] was used as our estimate of $\sigma$ for healthy and diseased colonies of the different size classes (Table 1).

The probability of growing into the next size class $(\gamma)$ and that of retrogressing to a smaller one $(\rho)$ were estimated from the annual gain (for $\gamma$ ) or loss (for $\rho$ ) of live tissue area for each of the six size-health states by calculating the amount of years (T) it would take colonies to either gain or lose $500 \mathrm{~cm}^{2}$ using the colony size dynamics data of Toledo-Hernández et al. [7]. The reciprocal of $\mathrm{T}$ was used as an estimate of the probability of either growing into the next size class $(\gamma)$ or retrogressing $(\rho)$ [18]. Two out of 72 healthy colonies acquired disease during the study period and one out of 21 diseased colonies lost all visual signs of disease [7]. We used these as our estimates of $\alpha$ and $\beta$, respectively. Values of $\sigma, \alpha, \beta, \gamma$, and $\rho$ for the three size classes and two health states and the corresponding life cycle transitions are found in Table 2.

Although fecundity and early life cycle transitions have been estimated for some gorgonians [20], in G. ventalina this information is scarce; in lieu we used the recruitment data of Yoshioka [21]. Yoshioka [21] measured recruitment of gorgonians as a function of local adult abundance for a period of eight years in two localities in Puerto Rico. His data indicates a peak recruitment event of approximately of $1.3 \mathrm{G}$. ventalina recruits per $G$. ventalina adult, followed by a sevenfold reduction to baseline levels the following years. We use this estimate of peak recruitment (1.3) and the baseline level $(1.3 / 7=0.19)$ as the range of possible values that $F 2+$ F3 can attain, assuming that large colonies have twice the contribution of medium ones towards total recruitment.

\section{Results}

The area between the high and low recruitment trajectories in Figure 1 represents the possible annual asymptotic growth rates $(\lambda)$ that a sea fan population may exhibit as a function of disease incidence and recruitment given the considered parameter space. Our analysis predicts that populations with low persistent recruitment will go extinct irrespective of disease incidence (Figure 1). Notice that given low recruitment a disease-free population (incidence $=0$ ) will exhibit $\lambda$ of less than 1.0. On the other hand, the disease incidence threshold for population viability $(\lambda \geq 1)$ in populations with high recruitment is 0.12 . Notice, also, that recruitment has a large
TABLE 1: Annual survivorship estimates $(\sigma)$ of healthy $(h)$ and diseased $(d)$, small $(S)$, medium $(M)$, and large $(L)$ colonies of Gorgonia ventalina. Fate frequencies obtained from Toledo-Hernández et al. (2009) [7].

\begin{tabular}{lcccc}
\hline & $S_{h}$ & $S_{d}$ & $M_{h+d}$ & $L_{h+d}$ \\
\hline Survived & 22 & 7 & 24 & 14 \\
Died & 9 & 11 & 4 & 1 \\
Total & 31 & 18 & 28 & 15 \\
$\sigma$ & 0.7097 & 0.3889 & 0.8571 & 0.9333 \\
\hline
\end{tabular}

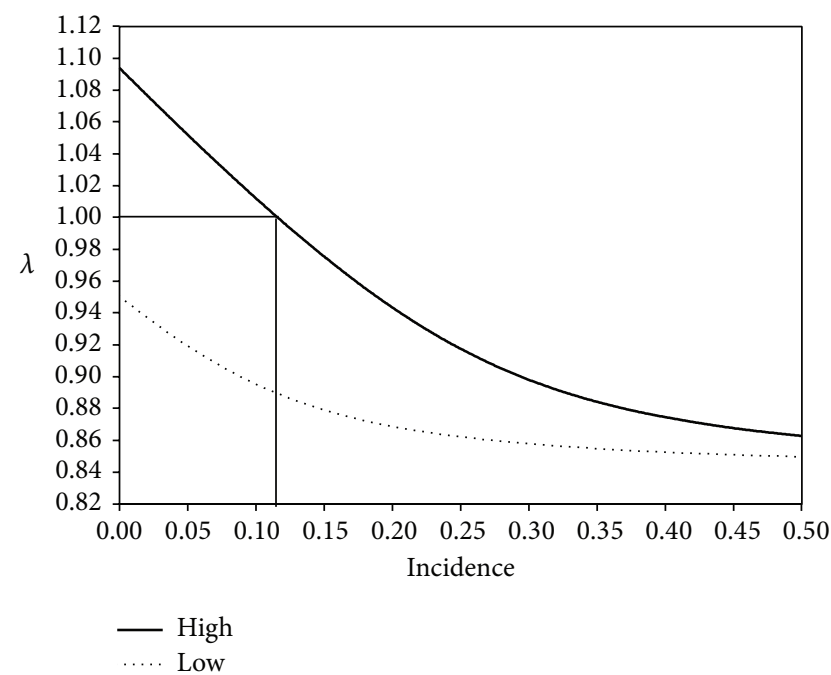

FIgURE 1: Asymptotic population growth rate $(\lambda)$ in the sea fan Gorgonia ventalina as a function of disease incidence for high (1.3 recruits/year/adult colony) and low (0.19 recruits/year/adult colony) levels of recruitment. Incidence threshold for population viability $(\lambda \geq 1.0)$ is shown.

effect on $\lambda$ for low levels of incidence, but as incidence increases, the effect of recruitment on $\lambda$ becomes negligible.

The stable size structure $(\mathbf{w})$ was calculated for three levels of incidence $(0,0.1$, and 0.2$)$ and for high (1.3 recruits per adult) and low (0.19 recruits per adult) recruitment (Figures 2(a) and 2(b)). Under zero incidence and high level of recruitment, small colonies dominate the population. The low relative abundance of medium and large colonies is the result of low transition rate of small colonies to medium ones. Under high recruitment and incidence greater than zero, the relative abundance of diseased colonies (i.e., prevalence) is similar among the three size classes. By contrast, under low recruitment, the relative abundance of diseased medium and large diseased colonies increases. Recruitment also has an effect on disease prevalence. For any given level of incidence, prevalence is higher in populations experiencing low recruitment, particularly in medium and large colonies, than in populations with higher recruitment. Disease also has an effect on the size structure of the population, decreasing the relative abundance of small colonies and increasing that of medium and large ones (Figure 2).

The elasticity analysis revealed that, for a healthy population (incidence $=0$ ) with high recruitment ( 1.3 recruits per 
TABLE 2: Estimates of life cycle transitions of the demographic model of Gorgonia ventalina presented in (1). The model considers three size classes (small, medium, and large) and two health states (healthy or diseased). Transition probabilities are a product of annual survivorship $(\sigma)$, growth $(\gamma)$, retrogression $(\rho)$, disease incidence $(\alpha)$, and healing $(\beta)$. See text for further explanations.

\begin{tabular}{|c|c|c|c|c|c|c|c|c|}
\hline State $(t)$ & State $(t+1)$ & Life cycle transition & $\sigma$ & $\gamma$ & $\rho$ & $\alpha$ & $\beta$ & Transition value \\
\hline$S_{h}$ & $S_{h}$ & $P 1_{h h}$ & 0.7097 & 0.1780 & & 0.0278 & & 0.5512 \\
\hline$S_{h}$ & $S_{d}$ & $P 1_{d h}$ & 0.7097 & 0.1780 & & 0.0278 & & 0.0158 \\
\hline$S_{h}$ & $M_{h}$ & $G 1_{h h}$ & 0.7097 & 0.1780 & & 0.0278 & & 0.1194 \\
\hline$S_{h}$ & $M_{d}$ & $G 1_{d h}$ & 0.7097 & 0.1780 & & 0.0278 & & 0.0034 \\
\hline$S_{d}$ & $S_{h}$ & $P 1_{\text {hd }}$ & 0.3889 & 0 & & & 0.0476 & 0.0168 \\
\hline$S_{d}$ & $S_{d}$ & $P 1_{d d}$ & 0.3889 & 0 & & & 0.0476 & 0.3361 \\
\hline$M_{h}$ & $M_{h}$ & $P 2_{h h}$ & 0.8571 & 0.7358 & 0.2345 & 0.0278 & & 0.0214 \\
\hline$M_{h}$ & $M_{d}$ & $P 2_{d h}$ & 0.8571 & 0.7358 & 0.2345 & 0.0278 & & 0.0050 \\
\hline$M_{h}$ & $L_{h}$ & $G 2_{h h}$ & 0.8571 & 0.7358 & 0.2345 & 0.0278 & & 0.6358 \\
\hline$M_{h}$ & $L_{d}$ & $G 2_{d h}$ & 0.8571 & 0.7358 & 0.2345 & 0.0278 & & 0.0182 \\
\hline$M_{h}$ & $S_{h}$ & $R 2_{h h}$ & 0.8571 & & 0.2345 & 0.0278 & & 0.2027 \\
\hline$M_{h}$ & $S_{d}$ & $R 2_{d h}$ & 0.8571 & & 0.2345 & 0.0278 & & 0.0058 \\
\hline$M_{d}$ & $M_{h}$ & $P 2_{h d}$ & 0.8571 & 0.1707 & 0.1707 & & 0.0476 & 0.0291 \\
\hline$M_{d}$ & $M_{d}$ & $P 2_{d d}$ & 0.8571 & 0.1707 & 0.1707 & & 0.0476 & 0.5563 \\
\hline$M_{d}$ & $L_{h}$ & $G 2_{h d}$ & 0.8571 & 0.1707 & & & 0.0476 & 0.0072 \\
\hline$M_{d}$ & $L_{d}$ & $G 2_{d d}$ & 0.8571 & 0.1707 & & & 0.0476 & 0.1445 \\
\hline$M_{d}$ & $S_{h}$ & $R 2_{h d}$ & 0.8571 & & 0.1707 & & 0.0476 & 0.0072 \\
\hline$M_{d}$ & $S_{d}$ & $R 2_{d d}$ & 0.8571 & & 0.1707 & & 0.0476 & 0.1445 \\
\hline$L_{h}$ & $L_{h}$ & $P 3_{h h}$ & 0.9333 & & 0.3446 & 0.0278 & & 0.5882 \\
\hline$L_{h}$ & $L_{d}$ & $P 3_{d h}$ & 0.9333 & & 0.3446 & 0.0278 & & 0.0168 \\
\hline$L_{h}$ & $M_{h}$ & $R 3_{h h}$ & 0.9333 & & 0.3446 & 0.0278 & & 0.3093 \\
\hline$L_{h}$ & $M_{d}$ & $R 3_{d h}$ & 0.9333 & & 0.3446 & 0.0278 & & 0.0088 \\
\hline$L_{d}$ & $L_{h}$ & $P 3_{h d}$ & 0.9333 & & 0.3089 & & 0.0476 & 0.0304 \\
\hline$L_{d}$ & $L_{d}$ & $P 3_{d d}$ & 0.9333 & & 0.3089 & & 0.0476 & 0.6076 \\
\hline$L_{d}$ & $M_{h}$ & $R 3_{\text {hd }}$ & 0.9333 & & 0.3089 & & 0.0476 & 0.0136 \\
\hline$L_{d}$ & $M_{d}$ & $R 3_{d d}$ & 0.9333 & & 0.3089 & & 0.0476 & 0.2716 \\
\hline
\end{tabular}

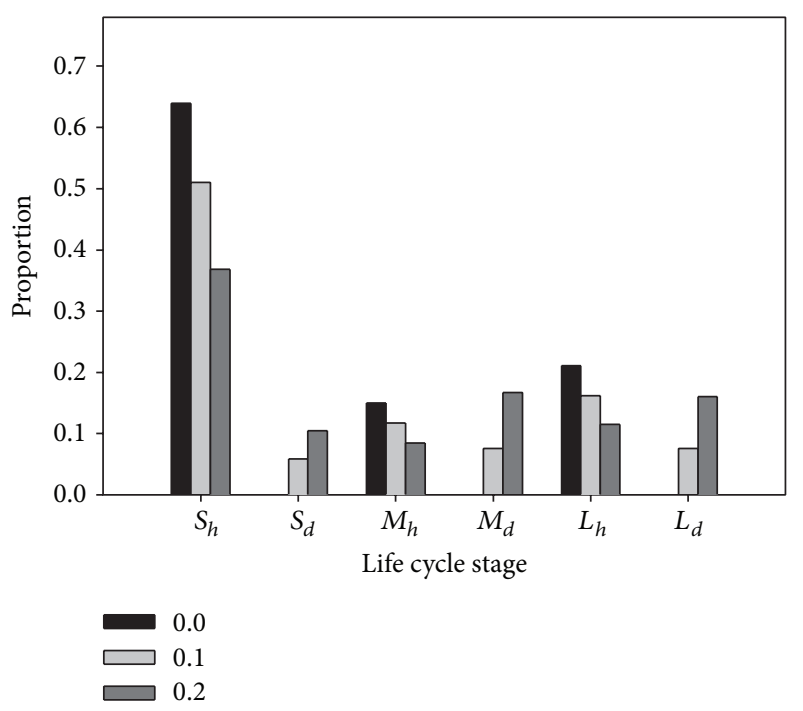

(a)
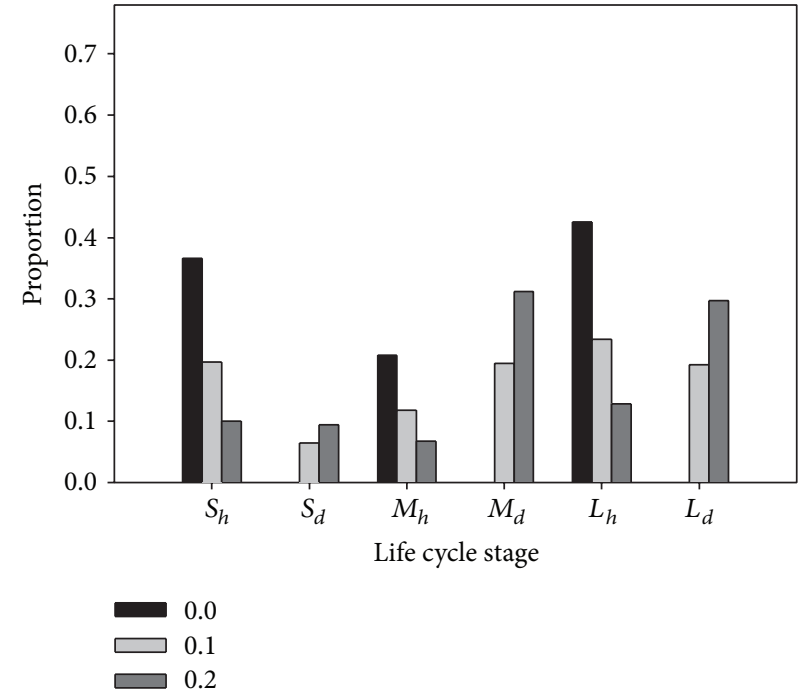

(b)

FIGURE 2: Stable stage distribution of Gorgonia ventalina colonies of three size classes (small $(S)$, medium $(M)$, and large $(L)$ ) and two health states (healthy $(h)$ and diseased $(d))$ for three levels of disease incidence $(0,0.10$, and 0.20$)$ under (a) high and (b) low recruitment. 


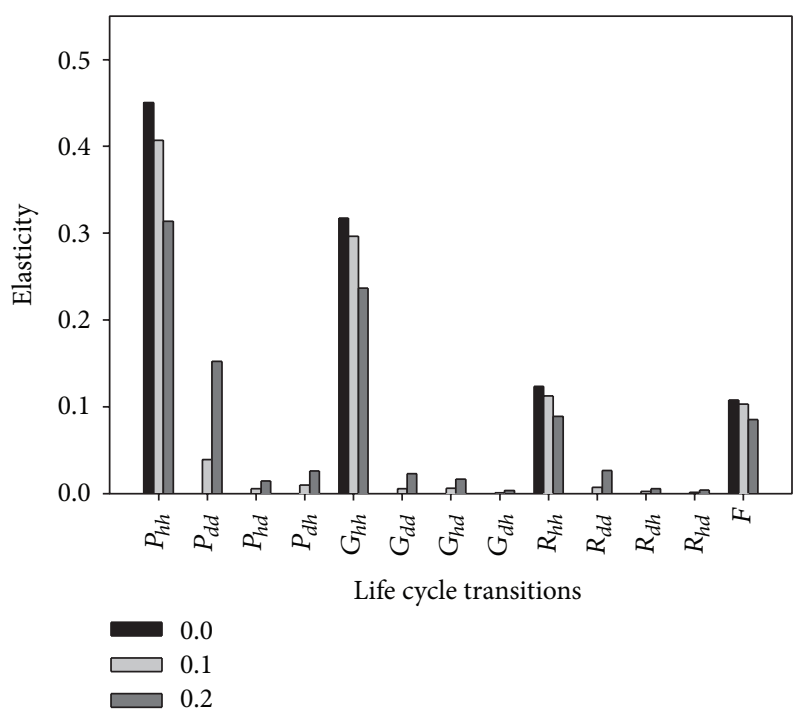

(a)

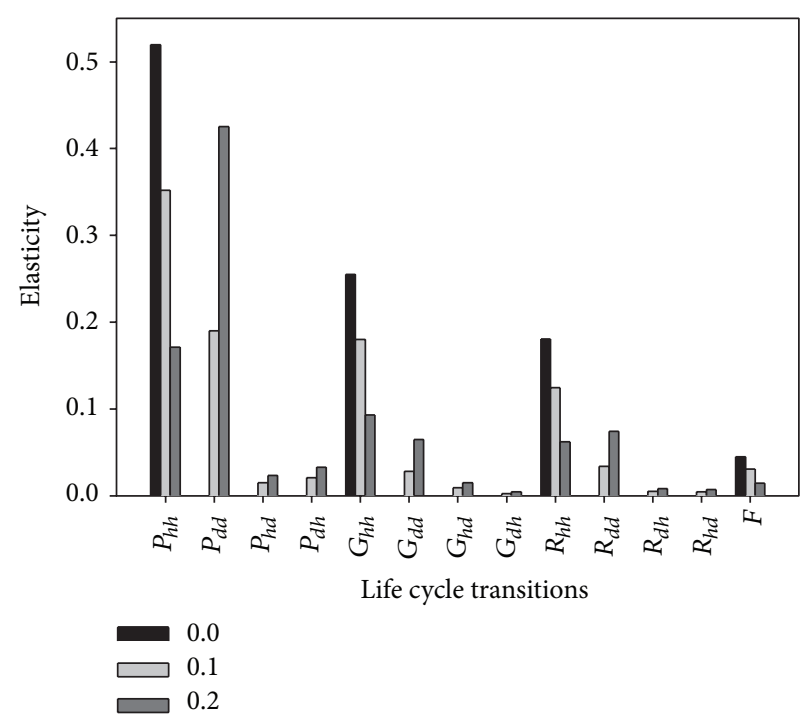

(b)

FIGURE 3: Elasticity of life cycle transitions of Gorgonia ventalina for three levels of disease incidence $(0,0.10$, and 0.20$)$ under (a) high and (b) low recruitment.

standing adult), survivorship is likely to have the strongest effect on $\lambda$, followed by growth, retrogression, and recruitment (Figure 3(a)). Low recruitment increases the relative importance of survivorship and retrogressions on $\lambda$ and decreases that of growth and recruitment (Figure 3(b)). Disease incidence lowers the relative importance of all transitions of healthy colonies, while increasing those of diseased ones. This is particularly evident with the survivorship of diseased colonies under low recruitment. Incidence also tends to increase the relative importance of survivorship relative to growth and fecundity, particularly for low recruitment.

\section{Discussion}

This study analyzes the effect of infection and recruitment rate on the viability of sea fan populations. Both of these processes can exhibit considerable spatial and temporal variation in corals. For example, gorgonians appear to be characterized by episodic recruitment during which populations can substantially increase in size [21], followed by long intervals of negligible recruitment during which populations are expected to be at or near steady state as a result of the high survival of adult colonies, or slowly declining due to high mortality of small colonies [22-24]. The dynamics of a coral epizootic can also show complex temporal and spatial dynamics $[10,16]$. As a result, the critical level of disease incidence above which colony abundance will start declining at a given locality is bound to be very variable. A coral population with high disease prevalence is not necessarily at risk if dying colonies are replaced by recruits, as demonstrated in some localities by sea fan populations impacted by disease in the Florida Keys [16]. We also know of coral species, such as Acropora palmata and A. cervicornis, which are characterized by low recruitment and in which diseases have significantly contributed to their collapse [25].

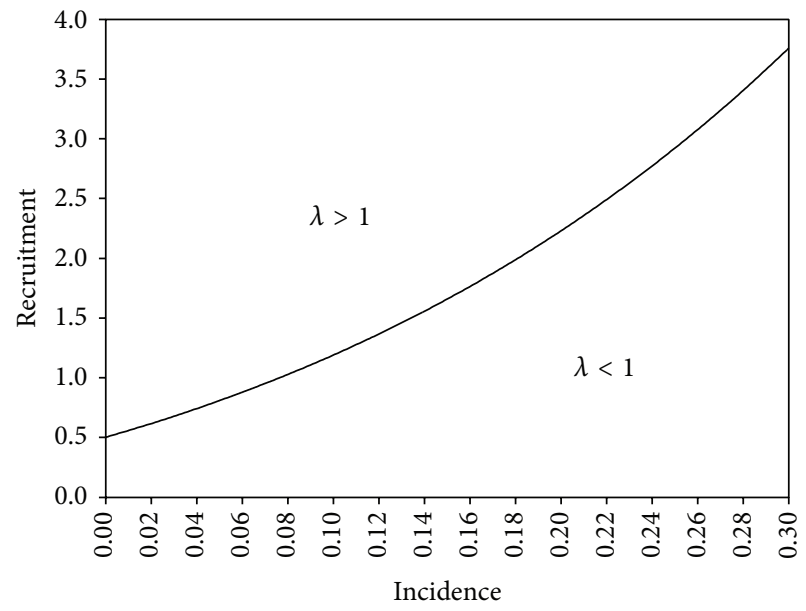

FIGURE 4: Annual number of Gorgonia ventalina recruits that a locality needs to receive (per standing adult) to maintain population equilibrium $(\lambda=1)$ as a function of disease incidence. Localities above the equilibrium threshold will exhibit population growth $(\lambda>$ $1)$, and those below it will decline $(\lambda<1)$.

It would be very useful to have an assessment tool which could be used to determine if a coral population impacted by disease is at risk $(\lambda<1)$ or not $(\lambda \geq 1)$. We used our model to answer this question for $G$. ventalina by determining the number of recruits necessary for a population to be at equilib$\operatorname{rium}(\lambda=1)$ as a function of disease incidence (Figure 4). Sea fan populations with levels of recruitment and incidence that fall above the equilibrium threshold would be viable while those falling below would be at risk (Figure 4). Our model predicts that populations with levels of incidence above 0.12 would cease to be viable even if experiencing high levels of recruitment (Figure 1). Even though an incidence of 0.12 is 
well above reported levels [6, 17], our analysis also predicts that aspergillosis may cause enough mortality to provoke population decline if recruitment is persistently low. In fact, the model predicts that even a healthy sea fan population (i.e., incidence $=0$ ) will decline if it receives less than 0.5 recruits per year per standing adult. Given the apparent episodic recruitment in gorgonians, even low levels of incidence will have the effect of accentuating population decline between recruitment episodes. This may lead to local extinction if the interval between recruitment events is too long.

Disease can affect the structure of a population, most noticeably the proportion of diseased colonies at a given time (i.e., prevalence) but also its size structure through differential mortality. Prevalence is generally understood to be directly proportional to incidence; our analysis, however, reveals that other processes such as mortality and recruitment may influence it as well. The model predicts that, with all other factors being equal, populations receiving fewer recruits should exhibit higher disease prevalence than populations with more recruitment. Thus, caution should be exercised when interpreting prevalence patterns in the absence of recruitment and mortality data. For example, an increase in prevalence in a given locality may be the result of a reduction in recruitment, and not to an increase in incidence, particularly in sea fans were medium and large colonies with lesions can persist for a long time [10]. Our analysis also reveals an effect of incidence on the size structure of sea fan populations. Increasing incidence lowers the relative abundance of small colonies, consequently increasing that of medium and large ones. This can be explained by the fact that aspergillosis can kill small colonies but has little impact on the survivorship of medium and large ones $[7,10]$. This also explains why prevalence is higher in medium and large colonies than in small ones.

Elasticity analysis is a prospective tool that provides insights regarding which life cycle transition could potentially have the greatest effect on population growth rate [18]. Our analysis identifies survivorship and growth of healthy colonies as the vital rates with the highest potential effect on population growth. This is consistent with the demography of other gorgonians $[22,24]$ and highlights the importance of survivorship of reproductive individuals given variable recruitment. Disease has the effect of increasing the relative impact of survivorship of diseased colonies on $\lambda$, particularly in small colonies and given low recruitment. This suggests selection for an immune response strong enough to contain and eradicate disease [26].

One of the main conclusions of the demographic analysis of $G$. ventalina by Bruno et al. [10] is that aspergillosis has very little impact on population growth rate and that the most important effect of this affliction on sea fan populations is to shift the size structure towards smaller size classes due to tissue necrosis. This is in contrast with the analysis we present in this study, as our analysis predicts a decline in population growth rate with increasing incidence that can range from $10 \%$ to $20 \%$ depending on the recruitment regime. The discrepancy between the models' predictions can be explained by the contrasting mortality patterns of the field data used to parameterize them. Both studies found no mortality that could be attributed to disease in medium or large colonies. However, our model does consider mortalities of $11 \%$ and $4 \%$ due to detachment of medium and large colonies, respectively, as reported by Toledo-Hernández et al. [7]. Bruno et al. [10], on the other hand, report no mortality due to detachment or to any other cause in medium or large colonies. This difference is of significance because both studies found survivorship (especially of large colonies) to be the transition with the highest elasticity. A population in which its reproductive individuals experience no mortality (or very close to zero for that matter) would be impervious to a reduction in recruitment, which is also another of their conclusions. However, if reproductive colonies are frequently removed from the population due to, for example, detachment, replacing them becomes important and, thus, a reduction in recruitment and/or in the survivorship of small colonies due to aspergillosis becomes critical. We simulated the former scenario in our model by decreasing detachment mortality and matching our recruitment estimate with theirs and were able to replicate their results (i.e., lack of response of population growth rate to disease incidence) as detachment mortality approaches zero.

This raises the interesting hypothesis that the demographic effect of disease on sea fan populations could be modulated by mortality due to abiotic processes, such as wave energy, which has also been recognized as an important source of mortality in other gorgonians [22]. The fact that Bruno et al. [10] did not observe detachment mortality can be explained by the fact that their study transects were established at depths between 6 and 8 meters and no high energy events such as hurricanes occurred during their monitoring period, whereas those of Toledo-Hernández et al. [7] were shallower (3-4 m) and at sites subjected to seasonal strong wave action (in particular long period waves resulting from winter storms in the North Atlantic). If probability of detachment is directly proportional to wave energy, we can expect sea fan aspergillosis to have a greater negative effect on populations at shallower depths, in the fore reef rather than the back reef areas, and during periods of high-energy events.

\section{Conflict of Interests}

The authors declare that there is no conflict of interests regarding the publication of this paper.

\section{Acknowledgments}

This study was financially supported by the NOAA-CRES (NOAA award NA170P2919) and Sea Grant (NOAA award NA16RG2278, Project R-92-1-04) programs of the UPR.

\section{References}

[1] T. P. Hughes, "Recruitment limitation, mortality, and population regulation in open systems: a case study," Ecology, vol. 71, no. 1, pp. 12-20, 1990.

[2] H. R. Lasker, K. Kim, and M. A. Coffroth, "Production, settlement, and survival of plexaurid gorgonian recruits," Marine Ecology Progress Series, vol. 162, pp. 111-123, 1998. 
[3] K. D. Lafferty, "The ecology of climate change and infectious diseases," Ecology, vol. 90, no. 4, pp. 888-900, 2009.

[4] S. Sokolow, "Effects of a changing climate on the dynamics of coral infectious disease: a review of the evidence," Diseases of Aquatic Organisms, vol. 87, no. 1-2, pp. 5-18, 2009.

[5] E. Weil, "Coral reef diseases in the wider Caribbean," in Coral Health and Diseases, E. Rosenberg and Y. Loya, Eds., pp. 35-68, Springer, New York, NY, USA, 2004.

[6] K. Kim, A. P. Alker, K. Shuster, C. Quirolo, and C. D. Harvell, "Longitudinal study of aspergillosis in sea fan corals," Diseases of Aquatic Organisms, vol. 69, no. 1, pp. 95-99, 2006.

[7] C. Toledo-Hernández, P. Yoshioka, P. Bayman, and A. M. Sabat, "Impact of disease and detachment on growth and survivorship of sea fans Gorgonia ventalina," Marine Ecology Progress Series, vol. 393, pp. 47-54, 2009.

[8] M. E. Brandt and J. W. McManus, "Dynamics and impact of the coral disease white plague: insights from a simulation model," Diseases of Aquatic Organisms, vol. 87, no. 1-2, pp. 117-133, 2009.

[9] S. H. Sokolow, P. Foley, J. E. Foley, A. Hastings, and L. L. Richardson, "Disease dynamics in marine metapopulations: modelling infectious diseases on coral reefs," Journal of Applied Ecology, vol. 46, no. 3, pp. 621-631, 2009.

[10] J. F. Bruno, S. P. Ellner, I. Vu, K. Kim, and C. D. Harvell, "Impacts of aspergillosis on sea fan coral demography: modeling a moving target," Ecological Monographs, vol. 81, no. 1, pp. 123139, 2011.

[11] G. W. Smith, L. D. Ives, I. A. Nagelkerken, and K. B. Ritchie, "Caribbean sea-fan mortalities," Nature, vol. 383, p. 487, 1996.

[12] D. M. Geiser, J. W. Taylor, K. B. Ritchie, and G. W. Smith, "Cause of sea fan death in the West Indies," Nature, vol. 394, no. 6689, pp. 137-138, 1998.

[13] C. Toledo-Hernández, A. Zuluaga-Montero, A. BonesGonzález, J. A. Rodríguez, A. M. Sabat, and P. Bayman, "Fungi in healthy and diseased sea fans (Gorgonia ventalina): is Aspergillus sydowii always the pathogen?" Coral Reefs, vol. 27, no. 3, pp. 707-714, 2008.

[14] A. Zuluaga-Montero, C. Toledo-Hernández, J. A. Rodríguez, A. M. Sabat, and P. Bayman, "Spatial variation in fungal communities isolated from healthy and diseased sea fans Gorgonia ventalina and seawater," Aquatic Biology, vol. 8, no. 2, pp. 151$160,2010$.

[15] I. Nagelkerken, K. Buchan, G. W. Smith et al., "Widespread disease in Caribbean sea fans: II. Patterns of infection and tissue loss," Marine Ecology Progress Series, vol. 160, pp. 255-263, 1997.

[16] K. Kim and C. D. Harvell, "The rise and fall of a six-year coralfungal epizootic," The American Naturalist, vol. 164, no. 5, pp. S52-S63, 2004.

[17] A. Zuluaga-Montero and A. M. Sabat, "Spatial variability of disease incidence and mortality in the sea fan Gorgonia ventalina in Puerto Rico (Alcyonacea: Goorgoniidae)," Revista de Biologia Tropical, vol. 60, no. 2, pp. 517-526, 2012.

[18] H. Caswell, Matrix Population Models: Construction, Analysis and Interpretation, MA Sinauer, Sunderland, UK, 2nd edition, 2001.

[19] L. E. Petes, C. D. Harvell, E. C. Peters, M. A. H. Webb, and K. M. Mullen, "Pathogens compromise reproduction and induce melanization in Caribbean sea fans," Marine Ecology Progress Series, vol. 264, pp. 167-171, 2003.

[20] C. Linares, R. Coma, S. Mariani, D. Díaz, B. Hereu, and M. Zabala, "Early life history of the Mediterranean gorgonian Paramuricea clavata: implications for population dynamics," Invertebrate Biology, vol. 127, no. 1, pp. 1-11, 2008.
[21] P. M. Yoshioka, "Variable recruitment and its effects on the population and community structure of shallow-water gorgonians," Bulletin of Marine Science, vol. 59, no. 2, pp. 433-443, 1996.

[22] H. R. Lasker, "Clonal propagation and population dynamics of a gorgonian coral," Ecology, vol. 71, no. 4, pp. 1578-1589, 1990.

[23] N. J. Gotelli, "Demographic models for Leptogorgia virgulata, a shallow-water gorgonian," Ecology, vol. 72, no. 2, pp. 457-467, 1991.

[24] C. Linares, D. F. Doak, R. Coma, D. Díaz, and M. Zabala, "Life history and viability of a long-lived marine invertebrate: the octocoral Paramuricea clavata," Ecology, vol. 88, no. 4, pp. 918928, 2007.

[25] R. B. Aronson and W. F. Precht, "White-band disease and the changing face of Caribbean coral reefs," Hydrobiologia, vol. 460, pp. 25-38, 2001.

[26] C. P. Ruiz-Diaz, C. Toledo-Hernández, A. M. Sabat, and M. Marcano, "Immune response to a pathogen in corals," Journal of Theoretical Biology, vol. 332, pp. 141-148, 2013. 

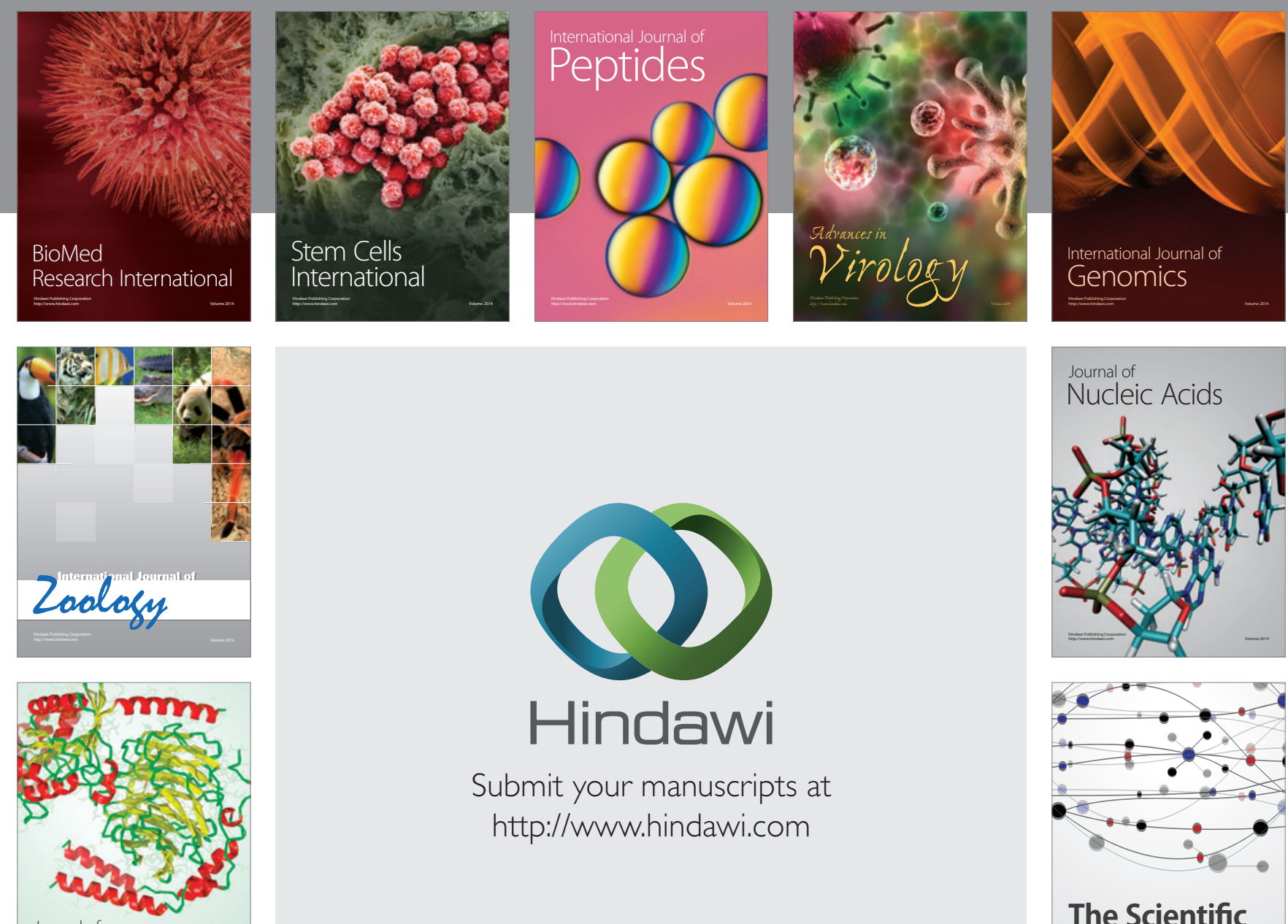

Submit your manuscripts at

http://www.hindawi.com

Journal of
Signal Transduction
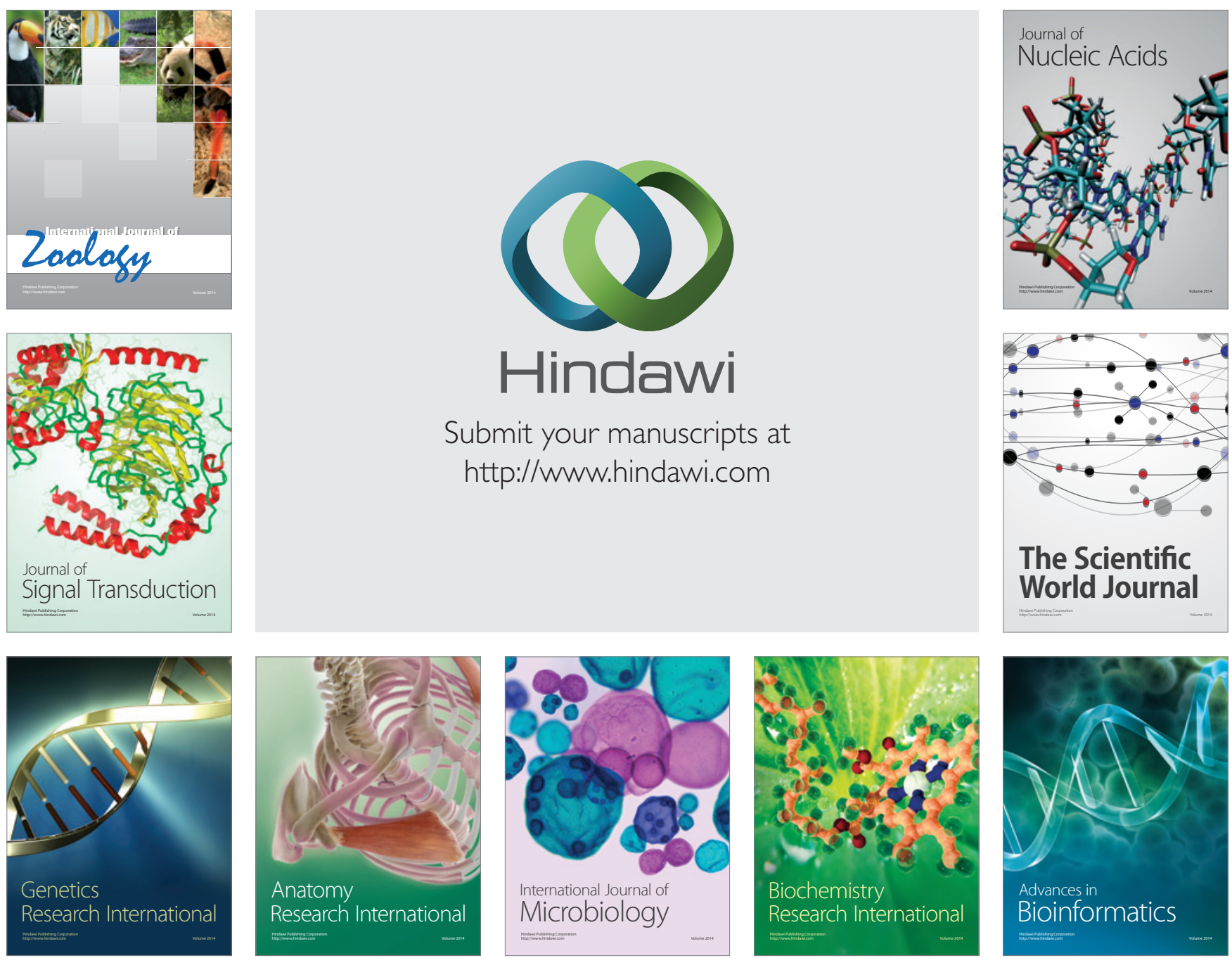

The Scientific World Journal
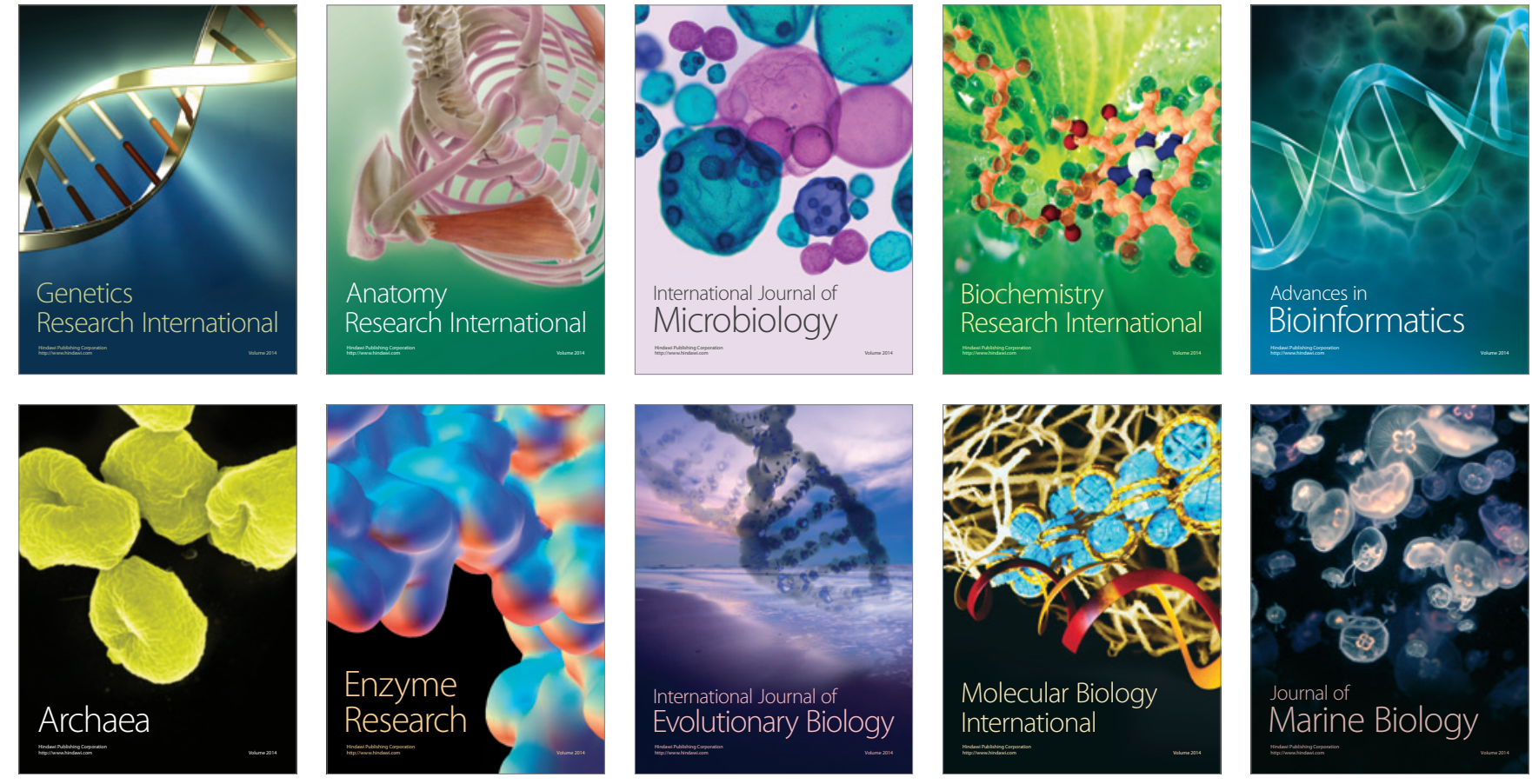